\title{
Comments on a Paper by Majorana Concerning Elementary Particles*
}

\author{
D. M. Fradkin \\ Department of Physics, Wayne State University, Detroit, Michigan
}

Received 13 October 1965, Published April 1966

\begin{abstract}
An early paper (1932) by Majorana, that has received but scant attention, is reexamined in light of later developments. This pioneering paper constructs a relativistically invariant theory of arbitrary spin particles, develops and utilizes infinite dimensional representations of the homogeneous Lorentz group, and provides a mass spectrum for elementary particles. The relevance of Majorana's approach and results to later and current research is explained.
\end{abstract}

Reprinted with permission from the AMERICAN JOURNAL OF PHYSICS, Volume 34, Issue 4, pp. 314-318. Copyright 1966, American Association of Physics Teachers.

We reproduce here the historical D. M. Fradkin 1966 paper whose role among the physicists of high energy was decisive; since then espressions like "Majorana mass", "Majorana spinors" and "Majorana neutrino" have become usual. The paper is based upon the work Teoria di Particelle con Momento Intrinseco Arbitrario, translated by Italiam from Edoardo Amaldi.

Ignazio Licata ${ }^{\dagger \ddagger}$

(C) Electronic Journal of Theoretical Physics. All rights reserved.

Keywords: Ettore Majorana, Elementary Particles

PACS (2006): 01.30.Tt, 01.65.+g, 11.30.-j

The paper "Teoria relativistica di particelle con momento intrinseco arbitrario" by Ettore Majoran (1932) [1,2] is remarkable for the following reasons : (i) it is apparently the first construction of a relativistically invariant theory of arbitrary half integer or integer spin particle; (ii) it is apparently the first recognition, development, and application of the infinite dimensional representations of the Lorentz group; (iii) it is a theory

\footnotetext{
* Reprinted with permission from the AMERICAN JOURNAL OF PHYSICS, Volume 34, Issue 4, pp. 314-318. Copyright 1966, American Association of Physics Teachers.

† Ignazio.licata@ejtp.info

$\ddagger$ ignazio.licata@ixtucyber.org
} 
that provides a mass spectrum for elementary particles. This pioneering paper, dealing with topics that in later years (and now!) have excited major interest, attracted little or no attention at its publication, and since then it has received only scant mention in several places [3-5]. The purpose of this present note is to rectify historical neglect by describing to a larger audience the content of Majorana's paper and placing his work in the the context of later research.

The starting point in Majorana's article is the single linear differential equation of Dirac form

$$
\left(\frac{W}{c}+\boldsymbol{\alpha} \cdot \mathbf{p}-\beta M c\right) \psi=0,
$$

Where $\boldsymbol{\alpha}$ and $\beta$ are numerical matrices, W is the energy, $\mathbf{p}$ is the momentum operator and $\psi$ is the multicomponent wavefunction. Unlike Dirac (1928) [6], he does not require the dispersion relation

$$
\left(\frac{W}{c}\right)^{2} \psi=\left[p^{2}+(M c)^{2}\right] \psi,
$$

To be satisfied for each component. As is well known, the imposition of both requirements, Eqs. (1) and (2), led Dirac [6] to certain commutation relation for $\boldsymbol{\alpha}$ and $\beta$-relations that Majorana does not assume. Many of the (finite dimensional) later higher spin theories [Petiau (1936), Duffin (1938), Kemmer (1939), and Fierz and Pauli (1939)] [3, 7-12] also have required the dispersion relation. In particular, the Dirac-Fierz -Pauli arbitrary spin theories [10-12] essentially factorize Eq. (2) into two coupled first-oder equations. For spin higher than one (spin value related to the number of components of $\psi$ ), these coupled first-order equations not only yield the dispersion relation, which describes the time development, but also yield additional restrictions on the wavefunction. These restrictions are known as subsidiary conditions. By insisting only on the linear form [Eq. (1)] but not the dispersion relation, one may avoid subjecting the wavefunction to subsidiary conditions. For finite representational theory, this point was explained years later by Bhabha [13]. An over- all brief summary of the invariant equations for finite dimensions is given by Umezawa (1956)[14].

At the time that Majorana wrote his article, it was an embarrassing fact that the original Dirac theory of the electron [6] introduced negative masses, i.e., that $\beta$ has eigenvalues \pm 1 . In order to avoid this, Majorana set for himself the following problem : Is it possible to have a relativistic invariant linear theory for which the eigenvalues of $\beta$ are all positive? In obtaining an affirmative answer, he was led to the infinite dimensional representations of the Lorentz group.

Majorana's arguments goes something like this: One of the conditions for relativistic invariance of the Dirac-form equation is that the Lagrangian density (which, in conjunction with the variational principle, can be used to derive this equation) must be invariant. A term of the density is $\psi^{*} \beta \psi$, so this is a relativistic invariant. But if $\beta$ has all positive eigenvalues, the quantity $\psi^{*} \beta \psi$ must be positive definite. Hence, it is possible to make a transformation $\psi \rightarrow \phi$ so that $\psi^{*} \beta \psi \rightarrow \phi^{*} \phi$ (this transformation is nonunitary and, in the representation where $\beta$ is diagonal is obviously accomplished by weighting the components of $\psi$ with the reciprocal square roots of the appropriate eigenvalues of $\beta$ ). 
In terms of $\phi$ then, relativistic invariance of the Lagrangian density implies that $\phi^{*} \phi$ is an invariant to all Lorentz transformations. This is certainly true if one can represent the Lorentz transformations in terms of unitary operators, i.e., if the generators of the infinitesimal Lorentz transformations have a specific Hermitian character.

With this in mind, Majorana displays the generators of the infinitesimal Lorentz transformations in the $4 \times 4$ space appropriate to operation on the coordinates, exhibits the generator commutation relations which must be satisfied in any representation, and then immediately gives the infinite dimensional Hermitian representations of these generators. In detail, an infinitesimal Lorentz transformation on the coordinates [15] is given by

$$
x_{\mu}^{\prime}=\left(\delta_{\mu \nu}+\xi_{\mu \nu}\right) x_{\nu}=\left(1-\frac{1}{2} i \xi_{\alpha \beta} I_{\alpha \beta}\right)_{\mu \nu} x_{\nu}
$$

Where here $\xi_{\mu \nu}$ are antisymmetric infinitesimals and $I_{\alpha \beta}$ represent the six independent group generators [16]. In this coordinate-base representation, the matrix components of the generators are

$$
\left(I_{\alpha \beta}\right)_{\mu \nu}=i\left(\delta_{\alpha \mu} \delta_{\beta \nu}-\delta_{\alpha \mu} \delta_{\beta \nu}\right)
$$

Defining the space-space generators $\mathbf{a}$ and the space-time generators $\mathbf{b}$ by the relations

$$
a_{i}=-\frac{1}{2} \varepsilon_{i j k} I_{j k}, b_{i}=i I_{i 4}
$$

One obtains by direct manipulation the commutation relations

$$
\begin{gathered}
{\left[a_{i}, a_{j}\right]=i \varepsilon_{i j k} a_{k},} \\
{\left[b_{i}, b_{j}\right]=-i \varepsilon_{i j k} a_{k},} \\
{\left[a_{i}, b_{j}\right]=i \varepsilon_{i j k} b_{k} .}
\end{gathered}
$$

For the infinite dimensional representations Majorana gives the matrix elements

$$
\begin{gathered}
\left(j, m\left|a_{1}-i \varepsilon a_{2}\right| j, m+\varepsilon\right)=[(j+\varepsilon m+1)(j-\varepsilon m)]^{\frac{1}{2}} \\
\left(j, m\left|a_{3}\right| j, m\right)=m
\end{gathered}
$$

and

$$
\begin{gathered}
\left(j, m\left|b_{1}-i \varepsilon b_{2}\right| j+\varepsilon \lambda, m+\varepsilon\right)=-\frac{1}{2} \lambda\left\{\left[(j+\lambda(m+\varepsilon)][j+1+\lambda(m+\varepsilon)\}^{\frac{1}{2}}\right.\right. \\
\left(j, m\left|b_{3}\right| j+\lambda, m\right)=\frac{1}{2}\left\{\left[j+m+\frac{1}{2}(\lambda+1)\right]\left[j-m+\frac{1}{2}(\lambda+1)\right]\right\}^{\frac{1}{2}}
\end{gathered}
$$


Here, $\varepsilon$ and $\lambda$ are independently \pm 1 , and non-indicated matrix element are zero. The index $\mathrm{m}$ ranges from $\mathrm{j}$ to $-\mathrm{j}$, and the range of $\mathrm{j}$ is either from 0 to $\infty$ by integer steps from $\frac{1}{2}$ to $\infty$ by integer steps.

Matrix elements identical with Majorana's infinite dimensional Lorentz representations [Eqs. (7) and (8)] were given earlier by Weyl (1928) [17] in connection with selection and intensity rules for the Schrödinger quantum mechanical problem of electric dipole transitions for an atom. How ever, it seems probable that Majorana derived these results himself. The fact that the three space-space generators a have the algebra of angular momenta suggests the angular momentum basis for their representation which Majorana employed [Eq. (7)]. The remaining three space-time generators $\mathbf{b}$ transform under spatial rotation like a three vector, so in effect they carry one unit of angular momentum. Thus, these generators have possible nonvanishing matrix elements between a basis with angular momentum $\mathrm{j}$ and bases $\mathrm{j}, \mathrm{j} \pm 1$ [18]. Consequently, the representation for all six generators involves all $\mathrm{j}$ values an integer apart (hence the fact that the representation is of infinite dimension ). The ones exhibited by Majorana are not the only infinite dimensional representations [18] nor does he make this claim, but he merely states (accurately) that they are the most simple.

The problem of unitary infinite-dimensional representations of the inhomogeneous Lorentz group was discussed years later by Wigner $(1939,1948)$ [4] from a different standpoint. Instead of proceeding from a definite wave equation and a postulated set of eigenfunctions on which the infinitesimal generators of the group act, Wigner assumed only the existence of a linear relativistically invariant manifold, i.e., a set of states which map into a superposition of themselves under the influence of a Lorentz transformation. This invariant -theoretic approach, though more general and certainly more rigorous than Majorana's development, gives somewhat less physical information (especially kinetic relation) and is of greater algebraic complexity. Following Wigner's first paper on the subject, the question of the infinite representation was investigated so little that in a later paper, reviving the study, Dirac (1945) [19] wrote: “.... The group has also some infinite representations which are unitary, These do not seem to have been studied much, in spite of their possible importance for physical application...." Dirac and subsequent authors, with the exception of Wigner [4] and Corson [6], make no reference to Majorana's pioneering work even though many of them employ essentially the same approach. Further detailed investigations of the infinite representations have been made by Gelfand and Naimark (1946) [20], Harish -Chandra (1947) [21], Bargmann (1947) [22], and Gelfand and Yaglom (1948) [23].

It is of interest to note that not only did Majorana give the infinite dimensional representations involving the series of integer $\mathrm{j}$ (special case of $\mathrm{j}$ bounded from below by 0 ) and the series of half -integer basis of angular momentum eigenfunctions of the Dirac electron theory. Moreover, Majorana recognized that this representations were special cases associated with a particular value (zero) of an invariant, namely $\mathbf{a} \cdot \mathbf{b}$, of the homogenous Lorentz group. In fact, there are two invariants characterizing a representation 
of the homogenous Lorentz group. These are the quantities

$$
\begin{aligned}
& \frac{1}{2} I_{\alpha \beta} I_{\alpha \beta}=(\mathbf{a} \cdot \mathbf{a}-\mathbf{b} \cdot \mathbf{b}), \\
& -\frac{1}{8} \varepsilon_{\mu \nu \alpha \beta} I_{\mu \nu} I_{\alpha \beta}=(\mathbf{a} \cdot \mathbf{b}),
\end{aligned}
$$

which can easily be shown to commute with all the generators a and $\mathbf{b}$. Consequently, in a given representation, these invariants have a fixed value and may be used to characterize the representation [21]. Thus, although Majorana recognized the role of one invariant with reference to a generalization of his results, he did not pursue the question of the group invariants to the extent of exhausting the possibility for generalization.

After Majorana developed the Hermition infinite dimensional representations of the Lorentz transformation generators, he went back to the Lagrangian density expressed in terms of the wavefunction $\phi$, and again insisted on invariance of the density under Lorentz transformations. This invariance requirement reduces to the insistence that the wave equation [24]

$$
\left(\gamma_{\mu} p_{\mu}^{\prime}-M c\right) \phi^{\prime}\left(x^{\prime}\right)=0
$$

be form invariant under the simultaneous transformations

$$
\begin{gathered}
\phi^{\prime}\left(x^{\prime}\right)=\left\{\exp \left[-\frac{1}{2} i \xi_{\alpha \beta} I_{\alpha \beta}\right]\right\} \phi(x), \\
p_{\mu}^{\prime}=\left(\delta_{\mu \nu}+\xi_{\mu \nu}\right) p_{\nu} .
\end{gathered}
$$

From this requirement, it follows that

$$
\left[I_{\alpha \beta}, \gamma_{\pi}\right]=i\left(\delta_{\beta \pi} \gamma_{\alpha}-\delta_{\alpha \pi} \gamma_{\beta}\right)
$$

These commutation relations together with the previously given infinite dimensional representations of $I_{\alpha \beta}$ (in terms of $\mathbf{a}$ and $\mathbf{b}$ ) essentially determine the matrices $\gamma_{\pi}$.

Majorana gives the results

$$
\begin{gathered}
\left(j, m\left|\gamma_{4}\right| j, m\right)=-i\left(j+\frac{1}{2}\right), \\
\left(j, m\left|\gamma_{1}-i \varepsilon \gamma_{2}\right| j+\varepsilon \lambda, m+\varepsilon\right)=-\frac{1}{2} i \varepsilon\{[j+\lambda(m+\varepsilon)][j+1+\lambda(m+\varepsilon)]\}^{\frac{1}{2}}, \\
\left(j, m\left|\gamma_{3}\right| j+\lambda, m\right)=\frac{1}{2} i \lambda\left\{\left[j+m+\frac{1}{2}(\lambda+1)\right]\left[j-m+\frac{1}{2}(\lambda+1)\right]\right\}^{\frac{1}{2}},
\end{gathered}
$$

where again $\varepsilon$ and $\lambda$ are independently \pm 1 , and nonindicated matrix elements are zero.

Finally, Majorana transformed the wave equation written in terms of $\phi[\mathrm{Eq}$. (10) without the primes] into the Hamiltonian form written in terms of $\psi$ [Eq. (1)]. For this purpose, he utilized a nonunitary transformation

$$
\phi=T \psi,
$$

where

$$
(j, m|T| j, m)=\left(j+\frac{1}{2}\right)^{-\frac{1}{2}},
$$


nonindicated matrix elements being zero. In this way, he recovered Eq. (1), for which a and $\beta$ are given in terms of the $\gamma^{\prime}$ 's and $\mathrm{T}$ [Eqs. (13) and (15)] by the relations

$$
\begin{gathered}
\alpha_{i}=T \gamma_{i} T \\
\beta=T^{2}, \text { i.e. },(j, m|\beta| j, m)=\left(j+\frac{1}{2}\right)^{-1} .
\end{gathered}
$$

Thus, Majorana had solved the problem of obtaining an explicit form of a wave equation which was relativistically invariant and involved only positive energy eigenvalues.

The eigenvalues of $\beta$ in Majorana's infinite dimensional theory are $\left(j+\frac{1}{2}\right)^{-1}$, where $\mathrm{j}$ ranges over -all values in appropriate integer or half integer series. Consequently, the rest mass eigenvalues for the infinite number of eigenfunctions in this theory are $M\left(j+\frac{1}{2}\right)^{-1}$, where $\mathrm{M}$ is an assigned constant. Apparently, Majorana was not interested in the idea of a mass spectrum, and this disinterest was perhaps understandable in view of the limited number of elementary particles known at that time [25]. Instead, Majorana emphasized a particular eigenfunction corresponding to the rest -mass eigenvalue characterized by $\mathrm{j}=\mathrm{s}$ ( $\mathrm{s}$ fixed) and he disregarded the other eigenfunctions as as being unphysical. He admitted that sufficiently strong interactions would cause transitions to these other unphysical states, so consequently he restricted the domain of applicability of the theory to interactions sufficiently weak so as to cause no transitions. This line of argument is exactly the same as the one used in Dirac electron theory at the time to prohibit transitions to the (unphysical) negative energy states. Also, for the physical eigenfunction of rest mass $M\left(j+\frac{1}{2}\right)^{-1}$, Majorana demonstrated that in the nonrelativistic limit , all the components of the eigenfunction labeled by $\mathrm{j}$ different from $\mathrm{s}$ vanished by order $\mathrm{v} / \mathrm{c}$ or grater. Again, this demonstration is parallel to the Dirac electron theory for which the "small" components of the positive energy eigenfunction vanish in the nonrelativistic limit [26]. Thus in the nonrelativistic limit, the infinite dimensional eigenfunction has only $2 \mathrm{~s}+1$ nonvanishing components in agreement with the ad hoc Pauli modification of Schrödinger quantum mechanics. To have only $2 \mathrm{~s}+1$ components in the nonrelativistic was one of the stated aims of Majorana's theory. Moreover, this fact means that in the rest system, The wavefunction transformed like an irreducible finite representation of the rotation group corresponding to spin s, and since spin is essentially defined by rest-system properties, the theory of Majorana was indeed one for a single pure spin associated with his selected mass eigenvalue.

Majorana completed his article by mentioning the existence of imaginary mass eigenvalues in the theory, developing the expression for the eigenfunctions in a plane wave state, and discussing the incorporation of an electromagnetic interaction by means of the usual replacement $p_{\mu} \rightarrow p_{\mu}-(e / c) A_{\mu}$. In this connection, he discussed how one may add a Pauli -type term (in the non-Hamiltonian form of the wave equation, essentially a term of the form $\gamma_{\mu} \gamma_{\nu} f_{\mu \nu}$ where $f_{\mu \nu}$ Is the electromagnetic field tensor) in order to provide for an anomalous magnetic moment [27]. His discussion of Pauli's procedure involves the only reference to published work that Majorana makes in his article, and even this is indirect as he refers to Pauli as cited by Oppenheimer! [28]. 
One may speculate why this article by Majorana occasioned little or no comment, and practically disappeared into the archives. First of all, just shortly before its publication, the positron was discovered [25] and this invested the negative mass eigenvalues (reinterpreted) of the Dirac electron theory with physical significance. Consequently, a theory which had mass eigenvalues of only one sign and did not incorporate the feature of antiparticles held little appeal. Furthermore, second quantization showed how to deal with transitions between different mass eigenvalues, so the weak field limit restriction was lifted. This meant that all of the mass eigenstates of Majorana's theory were accessible. But his mass spectrum was of the form $M\left(s+\frac{1}{2}\right)^{-1}$, which says that the higher the spin, the lower the mass. This relation was undesirable since it indicated that higher spin particles should be the more stable ones. Here, again, any interest in Majorana's theory would be inhibited.

Also, the infinite dimensional representations of the Dirac matrices are unwieldy and this is a disadvantage. Moreover, most physicists were unfamiliar with group theory, and its study was not much in fashion, so the exhibition of infinite dimensional representation of the homogeneous Lorents group did not find a very avid audience. Furthermore, the later development of the Dirac-Fierz-Pauli [10-12] theory which was based on a finite dimensional representation, had antiparticles, and proceeded from a factorized familiar dispersion relation may have saturated the interest of those pursuing the nonexsistent higher spin particles. Finally, the fact that Majorana's ,written in terse, condensed , somewhat cryptic style, was published in Italian in a journal not very widely read at the time [29] probably also contributed to its obscurity.

In recent years, Majorana's theory has been reconstructed, discussed, and generalized (always without reference to him). In this connection, one might mention the works of Gelfand and Yaglom (1948) [23], Ginzburg (1956) [30], Gelfand, Minlos, and Shapiro (1958) [31] and Naimark (1958) [32]. Some attempts have been made to combine a number of irreducible dimensional representations in order to produce a mass spectrum for which the values increase with increasing spin [33]

\section{Acknowledgment}

The author wishes to thank Professor E.Amaldi, at whose suggestion this review was undertaken, for introducing him to Majorana's paper and for many informative discussions. This work was initiated at the Istituto di Fisica, Universita di Rome, while the author was a 1964-65 NATO Fellow there. 


\section{References}

[1] E. Mojorana, Nuovo Cimento 9 , 335-344 (1932)

[2] The brilliant Italian physicist Ettore Majorana was born 5 August 1906 in Catania, Sicily and disappeared under unknown circumstances off coast of Naples on 26 March 1938. A fascinating account of Majorana's life and professional contributions has been given by E. Amaldi (to be published).

[3] N. Kemmer, "The Particle Aspect of Meson Theory", Proc. Roy. Soc. 173A, 91-116 (1939). Kemmer conjectured that existing higher spin theories, including Majorana's might be simplified by extensions of his (Kemmer) work.

[4] E. P. Wigner, " On Unitary Representations of the Inhomogeneous Lorentz Group", Ann. Math. (Princeton) 40, 149-204 (1939); "Relativistiche Wellengleichungen", Z. Physik 124, 665-684 (1948).

[5] E. M. Corson, Intoduction to Tensors, Spinors and Relativistic Wave -Equations (Blackie and Son, Ltd., Glasgow, 1953), Chap. 5, pp 140 and 199.

[6] P. A. M. Dirac, "The Quantum Theory of the Electron", Proc. Roy. Soc. (London) 117A, 610-624 (1928).

[7] G. Petiau, "Contribution á la Théorie des Equations d'Ondes corpusculaires" Thesis, Univ. Paris (1936) . Published in Mem. Acad. Sci. Roy. Belgique 16, No. 2, 1-116 (1936).

[8] R. J. Duffin, "On the Characteristic Matrices of Covariant" , Phys. Rev. 54, 1114 (1938)

[9] A. Proca , "Sur les equations fondamebtales des particules élémentaires", Compt Rend. 202, 1490-1492 (1936); "Sur la théorie ondulatoire des électrons positives et négatifs" J. Phys. Radium 7, 347-353 (1936).

[10] P. A. M. Dirac , "Relativistic Wave Equations", Proc. Roy. Soc. (London) 155A, 447-459 (1936).

[11] M. Fierz, " ber die relativistische theorie Kraftefreier Teilchen mit beliebigem Spin", Helv. Phys. Acta 12, 3-37 (1939).

[12] M. Fierz and W. Pauli, "On Relativistic Wave Equations of Particles of Arbitrary Spin in an Electromagnetic Field" , Proc. Roy. (London) 173A, 211-232 (1939).

[13] H. J. Bhabha, " Relativistic Wave Equations for the Elementary Particles", Rev. Mod. Phys. 17, 200-216 (1945). See also: E. Wild, "On First Order Wave Equations for Elementary Particles without Subsidiary Conditions", Proc. Roy. Soc. (London) 191A, 253-268 (1947); K. J. LeCouter, "The Structure of Linear Relativistic Wave Equations I, II", Proc. Roy. Soc. (London) 202A, 284-300, 394-407 (1950).

[14] H. Umezaawa, Quantum Field Theory (North-Holland Publishing Co., Amsterdam, 1956).

[15] Here, we deviate from Majorana's covariant -controvariant notation and take $x_{4}=$ $i c t$, thus avoiding the use of an explicit metric. In the following, Greek indices range from 1 to 4 , Latin from 1 to 3 , and summation convention on repeated indices is employed.

[16] As a consequence of the reality properties of the infinitesimals $\xi_{\mu \nu}$, a unitary representation here is one for which $I_{j k}$ is Hermitian and $I_{i 4}$ is anti-Hermitian. 
[17] H. Weyl, Gruppentheorie und Quantenmechanik (S. Hirzel Verlag, Leipzing, 1928). $1^{\text {st }}$ ed., P. 160. Since it is known that Majorana was very familiar with Weyl's Book (see Ref. 2), it is possible that Majorana took over Weyl's equations directly, having recognized that they also applied in his problem.

[18] The fact that the matrix elements of the b's[Eq. (8)] do not connect bases with the same $\mathrm{j}$, but only connect with $\mathrm{j} \pm 1$, merly reflects the special cases discussed by Majorana which have $\mathrm{j}$ lower bounds of 0 or $\frac{1}{2}$.

[19] P. A. M. Dirac, "Unitary Representations of the Lorentz Group", Proc. Roy. Soc. (London) 183A, 284-295 (1945).

[20] I. M. Gelfand and M. A. Naimark, "Unitary Representations of the Lorentz Group", J. Phys. (USSR) 10, 93-94 (1946).

[21] Harish -Chandra, "Infinite Irreducible Representations of the Lorentz Group", Proc. Roy. Soc. (London) 189A, 372-401 (1947).

[22] V. Bargmann, "Irreducible Unitary Representations of the Lorentz Group", Ann. Math. 48, 568-640 (1947).

[23] I. M. Gelfand and A. M. Yaglom, "General Relativistic -Invariant Equations and Infinite -Dimensional Representations of the Lorentz Group" (in Russian), Zh. Eksperim. I Teor. Fiz. 18, 707-733 (1948) [English transl.: TT-345, National Research Council of Canada (1953)].

[24] In Majorana's notation, $p_{4}=i W / c$ and $\gamma_{4}=-i \gamma_{0}$.

[25] The neutron had been discovered early in 1932 [J. Chadwick, "Possible Existence of a Neutron", Nature 129, 312 (1932); "The Existence of a Neutron", Proc. Roy. Soc. (London) 136A, 692-708 (1932)] and the positron had just been discovered [C. D. Anderson, "The Apparent Existence of Easily Deflectable Positives" , Science 76, 238-239 (1932)] several months before Majorana's article was published. However news of the latter event had not yet arrived in Rome when Majorana was preparing his paper.

[26] See, for example, A. Messiah, Quantum Mechanics (North- Holland Publishing Co., Amsterdam, 1962), Vol. 2, p933.

[27] This technique of Pauli is discussed and generalized by L. L. Foldy, "Electromagnetic Properties of Dirac Particles", Phys. Rev. 87, 688-693 (1952).

[28] J. F. Carlson and J. R. Oppenheimer, " The Impact of Fact Electrons and Magnetic Neutrons" , Phys. Rev. 41, 763-792 (1932). They refer to Pauli's 1931 Ann Arbor lectures.

[29] In 1932, Nuovo Cimento was not renowned Italian international journal that it is today. In fact, Science Abstracts (Section A, Physics), the English language abstracting service, did not abstract from Nuovo Cimento until 1946. Majorana's article was given a several line abstract by contemporary German abstract service [Physikalische Berichte, 1933 -I, P. 548] but the abstractor, whose major field was florescence of salts and crystal studies, failed to assess its significance or even mention the occurrence of the infinite dimensional representations.

[30] V. L. Ginzburg, “ On Relativistic Wave Equations with a Mass Spectrum”, Acta Phys. Polon. 15, 163-175 (1956).

[31] I. M. Gelfand, R. A. Minlos, and Z. Ya. Shapiro, Representations of the Rotation and Lorentz Group and their Applications (Pergamon Press, Inc., Oxford, 1963), translated by G. Cummins and T. Boddington from Russian (Fizmatgiz, Moscow, 1958). 
[32] M. Naimark, Linear Representations of the Lorentz Group (Pergamon Press, Inc., Oxford, 1964), translated by A. Swinfen and O. J. Marstrand from Russian (Fizmatgiz, Moscow, 1958).

[33] See English edition of Ref. 31, p. 340.

Reprinted with permission from the AMERICAN JOURNAL OF PHYSICS, Volume 34, Issue 4, pp. 314-318. Copyright 1966, American Association of Physics Teachers. 